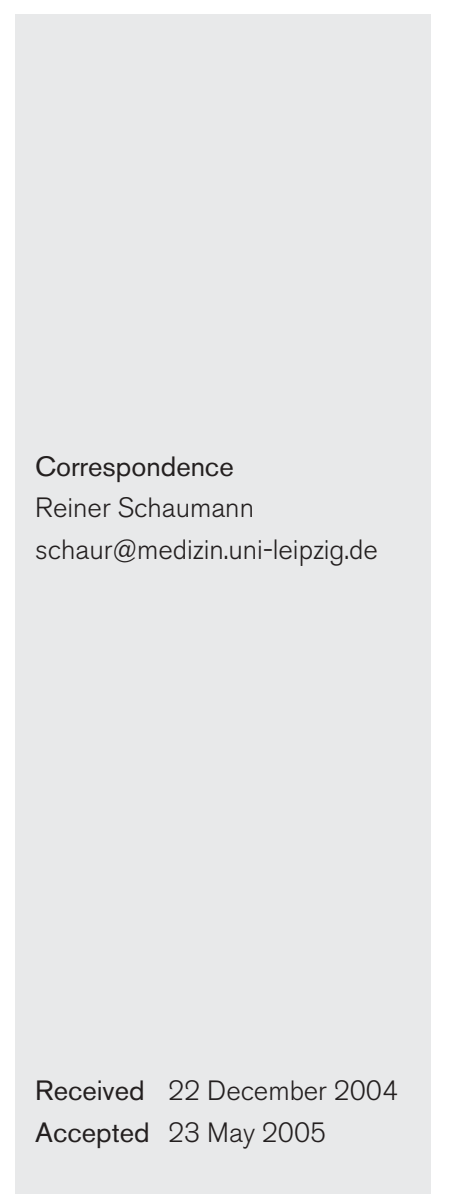

\title{
Activity of moxifloxacin against Bacteroides fragilis and Escherichia coli in an in vitro pharmacokinetic/ pharmacodynamic model employing pure and mixed cultures
}

\author{
Reiner Schaumann, ${ }^{1}$ Ellie J. C. Goldstein, ${ }^{2}$ Jochen Forberg ${ }^{3}$ \\ and Arne C. Rodloff ${ }^{1}$ \\ ${ }^{1}$ Institute for Medical Microbiology and Epidemiology of Infectious Diseases, University of Leipzig, \\ Leipzig, Germany \\ ${ }^{2}$ R. M. Alden Research Laboratories, Santa Monica, CA 90404, USA \\ ${ }^{3}$ Institute for Medical Informatics, Statistics and Epidemiology, University of Leipzig, Leipzig, Germany
}

\begin{abstract}
The objective of this study was to determine the pharmacodynamic (PD) activity of moxifloxacin against four selected Bacteroides fragilis strains (three strains with low MICs and one strain with a high MIC) and two Escherichia coli strains (one strain with a low MIC and one strain with a high MIC) in a pharmacokinetic (PK) in vitro model in pure cultures as well as in mixed cultures. PK/PD assays of moxifloxacin were carried out with an initial maximum concentration of $4.0 \mathrm{mg} \mathrm{I}^{-1}$ and a half-life of $13 \mathrm{~h}$. The $E$. coli strain with the low MIC was rapidly killed in both pure and mixed cultures in the in vitro PK/PD model, while the E. coli strain with the high MIC was not killed. None of the $B$. fragilis strains were rapidly killed in pure or mixed cultures. The bacterial numbers of the $B$. fragilis strains with low MICs were reduced by about one to two logs after $12 \mathrm{~h}$ in pure cultures. The presence of an $E$. coli strain with a low or a high MIC in the mixed culture reduced this effect even further.
\end{abstract}

\section{INTRODUCTION}

Obligately anaerobic bacteria comprise the largest part of the physiological flora on skin and mucous membranes of humans. Since they are opportunistic pathogens, they often participate in endogenous infections, causing mixed infections together with aerobic bacteria. Such infections (i.e. intra-abdominal) are burdened with high morbidity and mortality, and require treatment with antimicrobial drugs showing activity against both aerobic and anaerobic bacteria (Gorbach, 1994; Nathens \& Rotstein, 1996; Olsen et al., 1999).

Moxifloxacin is a quinolone that, like trovafloxacin and clinafloxacin, belongs to the fluoroquinolone group IV as defined by Naber \& Adam (1998). It has an antimicrobial activity against many Gram-positive and Gram-negative aerobic and anaerobic bacteria as well as atypical bacteria such as Chlamydia and Mycoplasma (Dalhoff et al., 1996; Bauernfeind, 1997; Goldstein et al., 1997; Edlund et al., 1998; Ackermann et al., 2000; Blondeau et al., 2000; Schaumann et al., 2000; Kleinkauf et al., 2001; Krasemann et al., 2001; Talan, 2001; Zhanel et al., 2002).

Abbreviations: PD, pharmacodynamic; PK, pharmacokinetic.
Several in vitro studies have indicated that moxifloxacin has good in vitro activity against important anaerobic bacteria especially Bacteroides species (Edlund et al., 1998; Ackermann et al., 2000). However, compared to the activities of garenoxacin, clinafloxacin, sitafloxacin and trovafloxacin, moxifloxacin more recently was the least active agent against 589 Bacteroides fragilis group isolates (Snydman et al., 2002). Furthermore, fluoroquinolone resistance among Bacteroides isolated in the USA has apparently markedly increased since 1994 (Golan et al., 2003). Hedberg \& Nord (2003) reported that antimicrobial resistance among $B$. fragilis group isolates in Europe is also increasing. Conversely, cidal moxifloxacin activity was found for respiratory pathogens (aerobes and anaerobes) even when sera were obtained $24 \mathrm{~h}$ after dosing. The results suggest that moxifloxacin may have clinical utility in the treatment of mixed aerobic/anaerobic respiratory tract infections (Stein et al., 2003b). In a recently published paper investigating serum bactericidal activity of moxifloxacin and gatifloxacin, Stein et al. (2003a) reported little or no serum bactericidal activity of either drug if the MICs of gatifloxacin were $\geqslant 2 \mathrm{mg} \mathrm{l}^{-1}$. However, moxifloxacin was found to be effective in vivo even against a $B$. fragilis strain with a high MIC level for moxifloxacin in an experimental animal model of severe mixed aerobic/anaerobic infection (Schaumann et al., 2004). 
The aim of the present study was to assess the killing activity of moxifloxacin in an in vitro pharmacokinetic/pharmacodynamic (PK/PD) model against four selected $B$. fragilis strains used previously in the in vivo experimental model (Schaumann et al., 2004). Since anaerobes are often present in mixed infections, kill kinetics were also established for mixed inocula employing the $B$. fragilis strains together with one of two different Escherichia coli strains, one strain with a low MIC for moxifloxacin and one with a high MIC.

\section{METHODS}

Bacterial strains. E. coli ATCC 25922, E. coli VA 6886 and different strains of B. fragilis (RMA 0309, RMA 5120, RMA 6791, WAL R 13267) were used. E. coli VA 6886 was isolated from bile at the Institute of Medical Microbiology of Leipzig, Germany. RMA 0309 and RMA 5120 were intra-abdominal isolates, RMA 6791 was a blood culture isolate and WAL R 13267 was a clinical isolate of unknown origin.

The B. fragilis strains and E. coli ATCC 25922 strain were characterized as follows as described previously (Schaumann et al., 2004): B. fragilis RMA 0309, enterotoxin negative, MIC for moxifloxacin $0.125 \mathrm{mg} \mathrm{l}^{-1}$; B. fragilis RMA 5120, enterotoxin negative, MIC for moxifloxacin $0 \cdot 125-0.38 \mathrm{mg}^{-1} ; B$. fragilis RMA 6791, enterotoxin positive, MIC for moxifloxacin $0 \cdot 25-0.5 \mathrm{mg} \mathrm{l}^{-1}$; B. fragilis WAL R 13267 , enterotoxin positive, MIC for moxifloxacin $>32 \mathrm{mg} \mathrm{l}^{-1}$; E. coli ATCC 25922, MIC value for moxifloxacin $<0.03 \mathrm{mg} \mathrm{l}^{-1}$.

The MIC for the E. coli strain VA 6886 was established by broth microdilution technique according to DIN 58940-8 (Deutsches Institut für Normung e.V., 2000). The result was confirmed by E-test (AB BIODISK) according to the manufacturer's instructions, resulting in a MIC value of $>32 \mathrm{mg} \mathrm{l}^{-1}$.

The E. coli strains were grown on Endo agar (bioMérieux) and the $B$. fragilis strains were grown on Columbia agar (Oxoid) supplemented with $5 \%$ sheep blood (Oxoid), vitamin K1 (Sigma) and haemin (Serva Feinbiochemica). After incubation, B. fragilis strains and E. coli strains were harvested from the plates and suspended separately in Brucella broth (Becton Dickinson) supplemented with vitamin K1 and haemin, and incubated overnight at $37{ }^{\circ} \mathrm{C}$ under anaerobic and aerobic conditions, respectively. Then the suspensions were adjusted turbidimetrically to contain approximately $1.2 \times 10^{9}$ c.f.u. $\mathrm{ml}^{-1} \mathrm{~B}$. fragilis and approximately $1.5 \times 10^{8}$ c.f.u. $\mathrm{ml}^{-1}$ E. coli. Cultures for the experimental model were set up as described below and the numbers of bacteria were confirmed by appropriate plating.

Antimicrobial agent. Moxifloxacin powder of known activity was kindly provided by Bayer Vital and suspended in distilled water.

Experimental model. In order to determine the pharmacodynamic activity of moxifloxacin all six strains were investigated in pure cultures as well as in mixed cultures. Cultures were set up in a final volume of $20 \mathrm{ml}$ of appropriately supplemented Brucella broth with approximately $2 \cdot 4 \times 10^{8}$ c.f.u. $\mathrm{ml}^{-1}$ B. fragilis or approximately $3 \times 10^{7}$ c.f.u. $\mathrm{ml}^{-1}$ E. coli or both, and an initial maximum concentration $\left(C_{\max }\right)$ of $4.0 \mathrm{mg}^{-1}$ moxifloxacin. The in vitro pharmacokinetic assays for moxifloxacin were carried out over $12 \mathrm{~h}$ with $t_{1 / 2}$ of $13 \mathrm{~h}$ according to the equation $C_{t}=C_{0} \times \mathrm{e}^{-k_{\mathrm{el}} \times{ }^{t}}\left(C_{t}=\right.$ concentration of moxifloxacin at a given point in time $(t) ; C_{0}=$ initial concentration of moxifloxacin). The elimination rate constant $\left(k_{\mathrm{el}}\right)$ was calculated using the equation $k_{\mathrm{el}}=\ln 2 / t_{1 / 2}$.

An observation time period of $12 \mathrm{~h}$ was chosen according to the half-life of moxifloxacin and the dosing interval suggested for intra-abdominal infections. We used a $C_{\max }$ concentration for moxifloxacin of $4 \mathrm{mg}^{-1}$ equal to total concentration since protein binding of moxifloxacin probably occurs in culture media as well. High inocula of approximately $2.4 \times 10^{8}$ c.f.u. $\mathrm{ml}^{-1}$ B. fragilis and approximately $3 \times 10^{7}$ c.f.u. $\mathrm{ml}^{-1}$ E. coli were used since abscesses contain a large number of bacteria (Stearne et al., 2001 \& 2002).

The PK/PD model was established by adding appropriate amounts of supplemented Brucella broth every $30 \mathrm{~min}(540-980 \mu \mathrm{l})$, resulting in a final volume of $36 \cdot 25 \mathrm{ml}$ and a final moxifloxacin concentration of $2 \cdot 11 \mathrm{mg} \mathrm{l}^{-1}$ after $12 \mathrm{~h}$. At $30 \mathrm{~min}$ intervals samples $(20 \mu \mathrm{l})$ were taken and diluted aliquots were plated on Endo agar as well as on supplemented Columbia agar. The experiments were carried out in an anaerobic chamber (Heraeus) containing $80 \% \mathrm{~N}_{2}, 15 \% \mathrm{CO}_{2}$ and $5 \% \mathrm{H}_{2}$ at $37^{\circ} \mathrm{C}$. However, the Endo agar plates were incubated under aerobic conditions for $24 \mathrm{~h}$. After incubation, bacterial colonies were counted and calculated to colony forming units per ml. The detection limit was $1 \times 10^{2}$ c.f.u. $\mathrm{ml}^{-1}$. In mixed cultures it was macroscopically possible to distinguish the colonies of $E$. coli from the colonies of $B$. fragilis strains growing on Columbia agar due to morphological criteria.

Statistical analysis. Mann trend test was used for testing for trends in a time-series of killing ratios (Hartung, 1999; Hollander \& Wolfe, 1999). A $P$ value of $<0.05$ was considered to be significant.

\section{RESULTS AND DISCUSSION}

For our PK/PD assay we employed a reasonably high initial concentration of moxifloxacin $\left(4 \mathrm{mg} \mathrm{l}^{-1}\right)$ that was reduced to approximately $2 \cdot 11 \mathrm{mg} \mathrm{l}^{-1}$ after $12 \mathrm{~h}$. In pure cultures the bacterial numbers of $B$. fragilis strains with low MICs $\left(\leqslant 0.5 \mathrm{mg} \mathrm{l}^{-1}\right)$ were moderately but significantly reduced (by about one to two logs) by moxifloxacin within $12 \mathrm{~h}$ (killing rates: RMA 0309, 99.3 \%; RMA 5120, 99.2 \%; RMA 6791, 99.2 \%; $P<0 \cdot 01$ ). For $B$. fragilis WAL R 13267 (MIC $\left.>32 \mathrm{mg} \mathrm{l}^{-1}\right)$ no reduction was observed $(P>0.05)$. Thus, none of the $B$. fragilis strains was effectively killed in pure culture (Fig. 1).

The E. coli ATCC 25922 strain (MIC $<0.03 \mathrm{mg} \mathrm{l}^{-1}$ ) was rapidly killed by moxifloxacin both in pure (data not shown) and mixed cultures (Fig. 2a), confirming a bactericidal effect against this strain $(P<0 \cdot 01$; killing rate within the first hour $>99.9 \%)$. As was to be expected, there was no bactericidal effect of moxifloxacin against the E. coli strain VA 6886 (MIC value: $>32 \mathrm{mg} \mathrm{l}^{-1}$ ) at the moxifloxacin concentrations employed here (Fig. 2b; $P>0.05$; data of pure culture not shown).

In mixed cultures employing E. coli strain ATCC 25922, with the low moxifloxacin MIC, the bacterial numbers of $B$. fragilis strains with low MICs were significantly reduced (killing rates: RMA 5120, 90.5 \%; RMA 6791, 91.8 \%; RMA $0309,99.8 \% ; P<0.01$; Fig. $2 \mathrm{a}$ ) and the growth rate of the $B$. fragilis strain with a high MIC (WAL R 13267) was significantly lower compared to the control without moxifloxacin $(P<0 \cdot 01)$. Nevertheless, in the presence of the $E$. colis strain ATCC 25922 the moderate activity of moxifloxacin against the anaerobes (except strain RMA 0309) was significantly reduced as compared to single anaerobic cultures $(P \leqslant 0 \cdot 01)$. In mixed cultures containing the $E$. coli strain VA 6886 and the B. fragilis strains with low MICs, the bacterial 

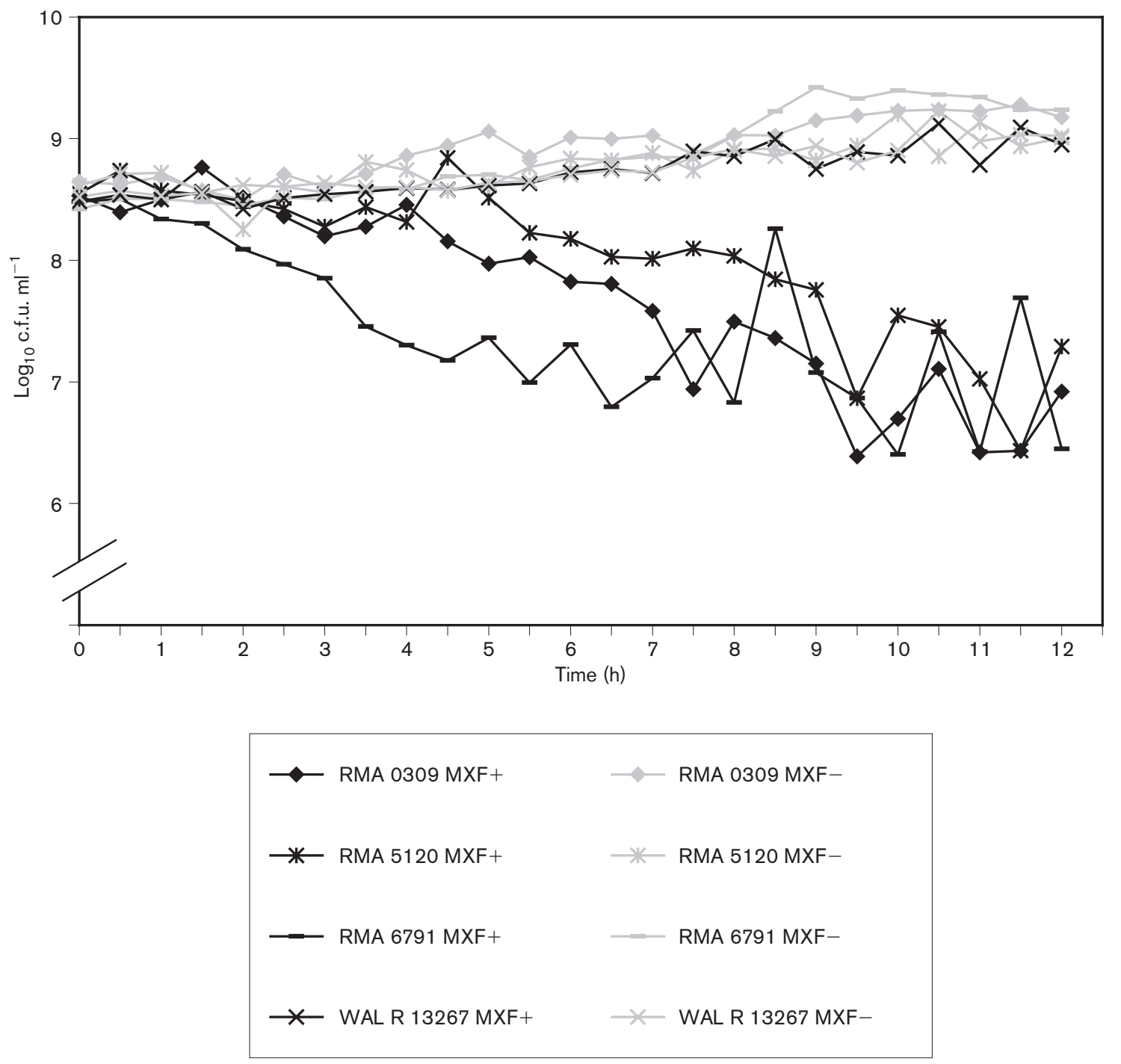

Fig. 1. Activity of moxifloxacin against four different strains of $B$. fragilis tested in the in vitro PK/PD model in pure cultures with and without moxifloxacin. MXF+, with moxifloxacin; MXF-, control without moxifloxacin. Detection limit: $10^{2}$ c.f.u. $\mathrm{ml}^{-1}$.

numbers of these bacteria were again significantly reduced compared to the control (killing rates: RMA 0309, 72.2\%; RMA 5120, 82.7 \%; RMA 6791, 88.2\%; $P<0.01$; Fig. 2b) but significantly less reduced if compared to pure cultures with moxifloxacin $(P<0 \cdot 01)$. Comparing the effects of the two $E$. coli strains on the anaerobes a significant difference in killing was only found for $B$. fragilis RMA $0309(P<0.01)$. In addition, the growth of the $B$. fragilis WAL R 13267 strain was significantly lower in the presence of the E. coli VA 6886 strain when compared to pure culture or to the mixed culture with E. coli ATCC $25922(P<0 \cdot 01)$. However, the growth of the $B$. fragilis WAL R 13267 strain was not reduced by moxifloxacin compared to the initial inoculum.

In contrast to the moderate activity of moxifloxacin investigated here using the in vitro PK/PD model, moxifloxacin has been shown to be as efficacious as imipenem/cilastatin in the treatment of severe systemic mixed aerobic/anaerobic infection in mice using the same B. fragilis strains and E. coli strain ATCC 25922 (Schaumann et al., 2004). Earlier observations of Onderdonk et al. (1976) suggested that E. coli is primarily responsible for the lethal effects in the animals. Therefore, the results of the PK/PD model support those previous observations since in the in vitro $\mathrm{PK} / \mathrm{PD}$ model, moxifloxacin was fully bactericidal against the E. coli strain ATCC 25922. The presence of the E. coli ATCC 25922 and VA 6886 strains reduced the killing activity of moxifloxacin against the $B$. fragilis strains investigated in the in vitro $\mathrm{PK} / \mathrm{PD}$ model. This observation is supported by earlier results obtain with trovafloxacin (Stearne et al., 2001). Furthermore, protection of $B$. fragilis against the activity of metronidazole by Enterococcus faecalis has also been observed (Nagy \& Földes, 1991). Nagy \& Földes (1991) reported that metronidazole was inactivated by a cell extract of E. faecalis. 

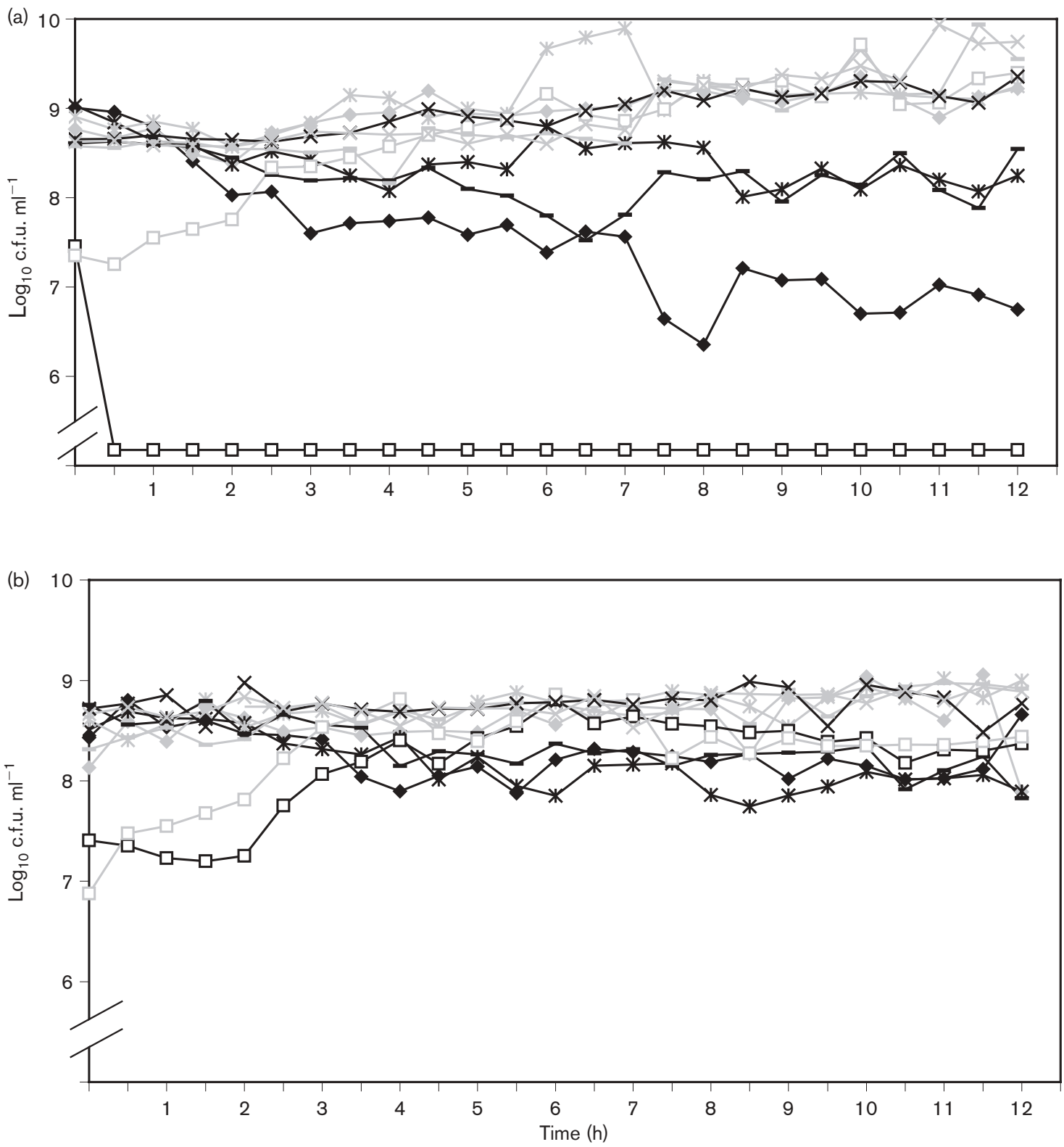

\begin{tabular}{|c|c|c|c|}
\hline$\leadsto$ RMA 0309 MXF+ & $\multimap$ RMA 0309 MXF- & $\rightarrow$ RMA $5120 \mathrm{MXF}+$ & * RMA 5120 MXF- \\
\hline — RMA $6791 \mathrm{MXF}+$ & $=$ RMA 6791 MXF- & $\rightarrow \square-E C M X F+$ & $-\square-E C M X F-$ \\
\hline$\star$ WAL R $13267 \mathrm{MXF}+$ & $\lessdot$ WAL R $13267 \mathrm{MXF}-$ & & \\
\hline
\end{tabular}

Fig. 2. Activity of moxifloxacin against four different strains of $B$. fragilis tested in the presence of (a) E. colistrain ATCC 25922 (low MIC for moxifloxacin) and (b) E. colistrain VA 6886 (high MIC for moxifloxacin) in the in vitro PK/PD model. MXF+, with moxifloxacin; MXF-, control without moxifloxacin; EC, E. coli ATCC 25922 in (a) or E. coli VA 6886 in (b). Detection limit: $10^{2}$ c.f.u. ml $l^{-1}$.

However, inactivation of trovafloxacin by Enterococcus faecium was not described (Stearne et al., 2001).

The reason for the different killing rates of moxifloxacin against the $B$. fragilis strains with low MICs investigated in our PK/PD model in mixed cultures is not yet understood. Stearne et al. (2001) discussed whether the emergence and selection of trovafloxacin-resistant mutants, with possible transfer of this resistance to $B$. fragilis in mixed infections, could account for the protection against the activity of the 
antimicrobial agent. However, further investigations would be required to confirm this hypothesis. Because of the results reported here, the clinical study data obtained for moxifloxacin treatment of patients with intra-abdominal infections need intensive scrutiny.

\section{ACKNOWLEDGEMENTS}

This study was supported by a grant from Bayer Vital GmbH, Leverkusen, Germany. This work was presented in part at the 43rd Interscience Conference on Antimicrobial Agents and Chemotherapy, Chicago, IL, 14-17 September 2003.

\section{REFERENCES}

Ackermann, G., Schaumann, R., Pless, B., Claros, M. C., Goldstein, E. J. C. \& Rodloff, A. C. (2000). Comparative activity of moxifloxacin in vitro against obligately anaerobic bacteria. Eur J Clin Microbiol Infect Dis 19, 228-232.

Bauernfeind, A. (1997). Comparison of the antibacterial activities of the quinolones Bay 12-8039, gatifloxacin (AM 1155), trovafloxacin, clinafloxacin, levofloxacin and ciprofloxacin. J Antimicrob Chemother 40, 639-651.

Blondeau, J. M., Laskowski, R., Bjarnason, J. \& Stewart, C. (2000). Comparative in vitro activity of gatifloxacin, grepafloxacin, levofloxacin, moxifloxacin and trovafloxacin against 4151 Gram-negative and Gram-positive organisms. Int J Antimicrob Agents 14, 45-50.

Dalhoff, A., Petersen, U. \& Endermann, R. (1996). In vitro activity of BAY 12-8039, a new 8-methoxyquinolone. Chemotherapy 42, 410-425.

Deutsches Institut für Normung e.V. (2000). Medizinische Mikrobiologie und Immunologie: Diagnostische Verfahren, 3rd edn. Berlin/Wien/ Zürich: Beuth.

Edlund, C., Sabouri, S. \& Nord, C. E. (1998). Comparative in vitro activity of BAY 12-8039 and five other antimicrobial agents against anaerobic bacteria. Eur J Clin Microbiol Infect Dis 17, 193-195.

Golan, Y., McDermott, L. A., Jacobus, N. V. \& 11 other authors (2003). Emergence of fluoroquinolone resistance among Bacteroides species. J Antimicrob Chemother 52, 208-213.

Goldstein, E. J. C., Citron, D. M., Hudspeth, M., Hunt Gerardo, S. \& Merriam, V. (1997). In vitro activity of Bay 12-8039, a new 8methoxyquinolone, compared to the activities of 11 other oral antimicrobial agents against 390 aerobic and anaerobic bacteria isolated from human and animal bite wound skin and soft tissue infections in humans. Antimicrob Agents Chemother 41, 1552-1557.

Gorbach, S. L. (1994). Antibiotic treatment of anaerobic infections. Clin Infect Dis 18 Suppl. 4, S305-S310.

Hartung, J. (1999). Statistik: Lehr- und Handbuch der angewandten Statistik, 12th edn. München/Wien/Oldenbourg: R. Oldenbourg Verlag.

Hedberg, M. \& Nord, C. E. on behalf of the ESCMID Study Group on Antimicrobial Resistance in Anaerobic Bacteria (2003). Antimicrobial susceptibility of Bacteroides fragilis group isolates in Europe. Clin Microbiol Infect 9, 475-488.

Hollander, M. \& Wolfe, D. A. (1999). Nonparametric Statistical Methods, 2nd edn. New York: Wiley.
Kleinkauf, N., Ackermann, G., Schaumann, R. \& Rodloff, A. C. (2001). Comparative in vitro activities of gemifloxacin, other quinolones, and nonquinolone antimicrobials against obligately anaerobic bacteria. Antimicrob Agents Chemother 45, 1896-1899.

Krasemann, C., Meyer, J. \& Tillotson, G. (2001). Evaluation of the clinical microbiology profile of moxifloxacin. Clin Infect Dis 32 Suppl. 1, S51-S63.

Naber, K. G. \& Adam, D. unter Mitwirkung einer Expertengruppe der Paul-Ehrlich-Gesellschaft für Chemotherapie e V. (1998). Einteilung der Fluorchinolone. Chemotherapie J 7, 66-68.

Nagy, E. \& Földes, J. (1991). Inactivation of metronidazole by Enterococcus faecalis. J Antimicrob Chemother 27, 63-70.

Nathens, A. B. \& Rotstein, O. D. (1996). Antimicrobial therapy for intraabdominal infection. Am J Surg 172 Suppl. 6A, 1S-6S.

Olsen, I., Solberg, C. O. \& Finegold, S. M. (1999). A primer on anaerobic bacteria and anaerobic infections for the uninitiated. Infection 27, 159-165.

Onderdonk, A. B., Bartlett, J. G., Louie, T., Sullivan-Seigler, N. \& Gorbach, S. L. (1976). Microbial synergy in experimental intraabdominal abscess. Infect Immun 13, 22-26.

Schaumann, R., Ackermann, G., Pless, B., Claros, M. C., Goldstein, E. J. C. \& Rodloff, A. C. (2000). In vitro activities of fourteen antimicrobial agents against obligately anaerobic bacteria. Int J Antimicrob Agents 16, 225-232.

Schaumann, R., Blatz, R., Beer, J., Ackermann, G. \& Rodloff, A. C. (2004). Effect of moxifloxacin versus imipenem/cilastatin treatment on the mortality of mice infected intravenously with different strains of Bacteroides fragilis and Escherichia coli. J Antimicrob Chemother 53, 318-324.

Snydman, D. R., Jacobus, N. V., McDermott, L. A. \& 10 other authors (2002). In vitro activities of newer quinolones against Bacteroides group organisms. Antimicrob Agents Chemother 46, 3276-3279.

Stearne, L. E. T., Kooi, C., Goessens, W. H. F., Bakker-Woudenberg, I. A. J. M. \& Gyssens, I. C. (2001). In vitro activity of trovafloxacin against Bacteroides fragilis in mixed culture with either Escherichia coli or a vancomycin-resistant strain of Enterococcus faecium determined by an anaerobic time-kill technique. Antimicrob Agents Chemother 45, 243-251.

Stearne, L. E. T., Buijk, S. L., Mouton, J. W. \& Gyssens, I. C. (2002). Effect of a single percutaneous abscess drainage puncture and imipenem therapy, alone or in combination, in treatment of mixed-infection abscesses in mice. Antimicrob Agents Chemother 46, 3712-3718.

Stein, G. E., Schooley, S. \& Kaatz, G. W. (2003a). Serum bactericidal activity of the methoxyfluoroquinolones gatifloxacin and moxifloxacin against clinical isolates of Staphylococcus species: are the susceptibility breakpoints too high? Clin Infect Dis 37, 1392-1395.

Stein, G. E., Schooley, S., Tyrrell, K. L., Citron, D. M. \& Goldstein, E. J. C. (2003b). Bactericidal activities of methoxyfluoroquinolones gatifloxacin and moxifloxacin against aerobic and anaerobic respiratory pathogens in serum. Antimicrob Agents Chemother 47, 1308-1312.

Talan, D. A. (2001). Clinical perspectives on new antimicrobials: focus on fluoroquinolones. Clin Infect Dis 32 Suppl. 1, S64-S71.

Zhanel, G. G., Ennis, K., Vercaigne, L., Walkty, A., Gin, A. S., Embil, J., Smith, H. \& Hoban, D. J. (2002). A critical review of the fluoroquinolones: focus on respiratory infections. Drugs 62, 13-59. 